\title{
Ginsenoside Rp1 Exerts Anti-inflammatory Effects via Activation of Dendritic Cells and Regulatory T Cells
}

\author{
Jingyu Bae ${ }^{1}$, Jihye Koo ${ }^{1}$, Soochan Kim ${ }^{1}$, Tae-Yoon Park ${ }^{2}$, and Mi-Yeon Kim ${ }^{1 *}$ \\ ${ }^{1}$ Department of Bioinformatics and Life Science, College of Natural Sciences, Soongsil University, Seoul 156-743, Korea \\ ${ }^{2}$ Department of Biotechnology, College of Life Science and Biotechnology, Yonsei University, Seoul 120-749, Korea
}

Ginsenoside Rp1 (G-Rp1) is a saponin derivate that provides anti-metastatic activities through inhibition of the NF- $\mathrm{B}$ pathway. In this study, we examined the effects of G-Rp1 on regulatory T cell (Treg) activation. After treatment of splenocytes with G-Rp1, Tregs exhibited upregulation of IL-10 expression, and along with dendritic cells (DCs), these Tregs showed increased cell number compared to other cell populations. The effect of G-Rp1 on Treg number was augmented in the presence of lipopolysaccharide (LPS), which mimics pathological changes that occur during inflammation. However, depletion of DCs prevented the increase in Treg number in the presence of G-Rp1 and/or LPS. In addition, G-Rp1 promoted the differentiation of the memory types of CD4 ${ }^{+}$Foxp3 $^{+} \mathrm{CD} 2 \mathrm{~L}^{\text {low }}$ Tregs rather than the generation of new Tregs. In vivo experiments also demonstrated that Tregs and DCs from mice that were fed G-Rpl for $7 \mathrm{~d}$ and then injected with LPS exhibited increased activation compared with those from mice that were injected with LPS alone. Expression of TGF- $\beta$ and CTLA4 in Tregs was increased, and upregulation of IL-2 and CD80/ CD86 expression by DCs affected the suppressive function of Tregs through IL-2 receptors and CTLA4. These data demonstrate that G-Rp1 exerts anti-inflammatory effects by activating Tregs in vitro and in vivo.

Keywords: Panax ginseng, Regulatory T cell, Dendritic cell, Ginsenoside Rp1, Inflammation

\section{INTRODUCTION}

Panax ginseng has been used not only for medical purposes but also to boost health for a long time in Asian countries. In particular, red ginseng, which is steamed and sun-dried $P$. ginseng, has widely been utilized to boost the immune system. Many previous studies have indicated that red ginseng contains saponin components, such as ginsenoside Rh1 (G-Rh1), G-Rh2, G-Rg3, and G-Rg5, which all have anti-tumor activity in prostate cancer, ovarian cancer, and breast cancer [1-5]. Because these components are not stable, G-Rp1, which is a chemically stable component with ten-fold more potent anti-tumor activity than G-Rg3, has been derived from crude ginsenosides [6]. Kumar et al. [7] documented the

(c) This is an Open Access article distributed under the terms of the Creative Commons Attribution Non-Commercial License (http://creativecommons.org/licenses/by-nc/3.0/) which permits unrestricted non-commercial use, distribution, and reproduction in any medium, provided the original work is properly cited. safety and effects of G-Rpl on anti-tumor activity and demonstrated that G-Rp1-treated mice had a significant reduction in pathological symptoms in papillomagenesis. In addition, Park et al. [8] reported that mice with orallyadministered G-Rp1 displayed diminished pulmonary metastatic colony numbers in a mouse model of lung metastasis. Furthermore, G-Rp1 has been reported to inhibit pro-inflammatory cytokine production though inhibition of the nuclear factor kappa-light-chain-enhancer of activated B cells (NF- $\kappa \mathrm{B})$ pathway in macrophages $[9,10]$. Although several reports have shown that G-Rp1 possesses anti-tumor and anti-inflammatory activities, its function in cellular levels has not yet been studied.

Received 18 May. 2012, Revised 13 Jun. 2012, Accepted 16 Jun. 2012

*Corresponding author

E-mail: kimmy@ssu.ac.kr

Tel: +82-2-820-0458, Fax: +82-2-2-824-4383 
Foxp3-expressing regulatory $\mathrm{T}$ cells (Tregs) suppress the immune responses of other cells by producing the immunosuppressive cytokines IL-10 and TGF- $\beta$. The importance of Treg function has been shown in Foxp3deficient and IL-10-deficient mice, which developed severe autoimmune diseases [11-14]. In addition, Tregs are important for the suppression of inflammation at environmental interfaces, such as the lung and colon [15]. Dendritic cells (DCs) are professional antigen-presenting cells that present antigenic peptides on major histocompatibility class II molecules to helper T cells, leading to the adaptive immune response [16,17]. Recent studies showed that DCs activate Tregs directly and control Treg homeostasis in vivo $[18,19]$. In particular, a reduction in the numbers of DCs led to a loss of Tregs, and the remaining Tregs downregulated Foxp3 expression [18].

In this study, we investigated the effects of G-Rp1 on lymphocyte activation and cell numbers and Treg function in lipopolysaccharide (LPS)-induced inflammation. We found that G-Rp1 increases Treg numbers in concert with an increase in DC activation. Increased numbers of Tregs resulted from the conversion of memory type Tregs rather than the generation of new Tregs. In addition, in mice fed G-Rp1, we observed anti-inflammatory effects through DC and Treg activation.

\section{MATERIALS AND METHODS}

\section{Preparation of cells}

All experiments were performed in accordance with the approval of Soongsil University Institutional Animal Care and Use Committee. Cell suspensions were made from the spleens of 6-week-old C57BL/6 mice as described previously [20]. Briefly, spleens were cut into small fragments and then cultured in serum-free RPMI1640 medium with $5 \mathrm{mg} / \mathrm{mL}$ collagenase (Roche, Mammheim, Germany) for $45 \mathrm{~min}$ at $37^{\circ} \mathrm{C}$. Digested fragments were crushed between gauze. After depletion of red blood cells, the cell suspensions were cultured.

\section{Chemicals}

G-Rp1 was obtained from Ambo Institute (Seoul, Korea). G-Rp1; 3-O- $\beta$-D-glucopyranosyl $(1 \rightarrow 2)-\beta$-Dglucopyranosyl dammarane-3 $\beta, 12 \beta$-diol, was prepared in a large scale from G-Rg5 Rk1 by reduction with hydrogenation [6]. Splenocytes were cultured with 5, 10, or $20 \mu \mathrm{M}$ G-Rp1 and/or $1 \mu \mathrm{g} / \mathrm{mL}$ LPS (Sigma-Aldrich, St. Louis, MO, USA) for $2 \mathrm{~d}$, and/or $2 \mathrm{ng} / \mathrm{mL}$ TGF- $\beta$ (PeproTech, Rocky Hill, NJ, USA) for $3 \mathrm{~d}$.

\section{Depletion of dendritic cells}

To deplete CD $11 \mathrm{c}^{+} \mathrm{DCs}$, splenocytes were negatively enriched by using MACS anti-mouse CD11c microbeads according to the manufacturer's instructions (Miltenyi Biotec Ltd., Bergisch Glandbach, Germany).

\section{Flow cytometry}

Monoclonal antibodies (mAbs) for CD4 (clone RM45), CD11c (clone HL3), and CD45R/B220 (clone RA36B2) were purchased from BD Biosciences (San Jose, CA, USA), and mAbs for CD3 (clone 145-2C11), CD11c (clone N418), Foxp3 (clone 150D/E4), and IL-10 (clone JES516E3) were purchased from eBioscience (San Diego, CA, USA). Biotinylated mAbs against CD80 (clone 16-10A1), CD86 (clone GL1), CD62L (MEL-14), and CD25 (clone PC61.5) were obtained from eBioscience. As the secondstep staining reagents for biotinylated Abs, streptavidinphycoerythrin was purchased from BD Biosciences.

In order to detect Foxp3 and IL-10, cells were stained with surface Abs for CD3, CD4, CD11c, B220, and CD62L followed by intracellular Abs for Foxp3 or IL10 using a Foxp3 staining buffer set according to the manufacturer's instructions (eBioscience). In brief, after surface staining, Foxp3 fixation/permeabilization working solution was added to each tube and incubated for 30 $\min$ at $4^{\circ} \mathrm{C}$. Subsequently, permeabilization buffer was added to each tube and centrifuged for $5 \mathrm{~min}$. After that, the cells were stained for Foxp3 or IL-10.

\section{In vivo experiment}

Six-week-old C57BL/6 male mice, weighing $23 \pm 2 \mathrm{~g}$ were used for in vivo experiments. Seven mice of control group were injected with phosphate-buffered saline (PBS) and 6 mice of the second group were intraperitoneally injected with 5 ug LPS in PBS. Six mice of G-Rp1 group were orally administrated with G-Rp1 dissolved in $1 \%$ carboxymethylcellulose solution at $10 \mathrm{mg} / \mathrm{kg}$ body weight daily for $7 \mathrm{~d}$ before LPS injection.

\section{Regulatory $\mathrm{T}$ cell purification}

$\mathrm{CD} 4{ }^{+} \mathrm{CD} 25^{+}$cells were purified using mouse $\mathrm{CD} 4^{+} \mathrm{CD} 25^{+}$Treg isolation kit from MiltenyiBiotec. The isolation was performed in a two-step procedure. Firstly, non- $\mathrm{CD} 4^{+}$cells were indirectly magnetically labelled with a cocktail of biotin-conjugated Abs and incubated with anti-biotin microbeads. Labelled cells were depleted using LD columns (Miltenyi Biotec Ltd., Bergisch Glandbach, Germany). For the second step, pre-enriched $\mathrm{CD}^{+} \mathrm{T}$ cells were labelled with $\mathrm{CD} 25$ microbeads and positively selected using MS columns (MiltenyiBiotec). 


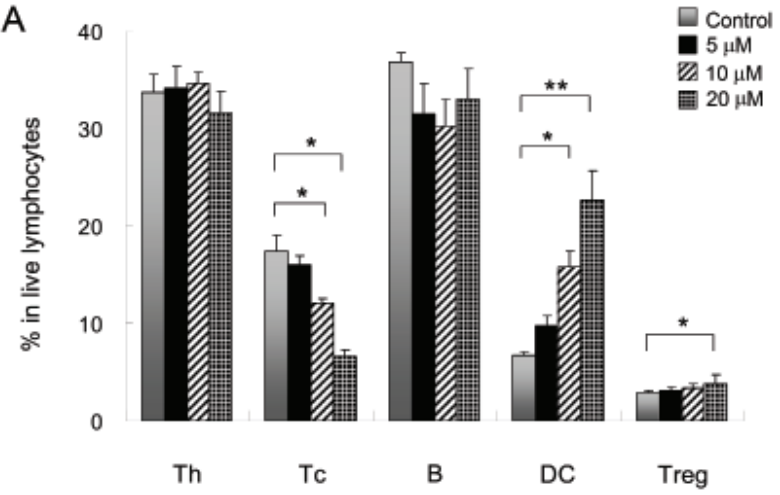

B

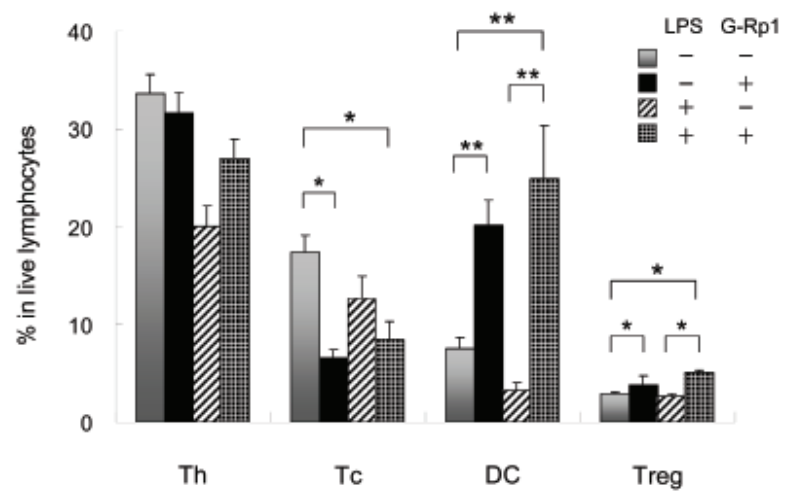

Fig. 1. Effect of ginsenoside Rp1 (G-Rp1) on slpenocytes. (A) Percentage of helper $T$ (Th) cells, cytotoxic $T$ (Tc) cells, B cells, dendritic cells (DCs), and regulatory T cells (Tregs). Splenocytes were cultured with 5,10 , or $20 \mu \mathrm{M}$ G-Rp1 for $2 \mathrm{~d}$, and analyzed using flow cytometry. (B) Percentage of Th cells, Tc cells, DCs, and Tregs in the presence or absence of G-Rp1 and LPS. Splenocytes were cultured with or without $20 \mu \mathrm{M} \mathrm{G-Rp} 1$ and $1 \mu \mathrm{g} / \mathrm{mL}$ lipopolysaccharide (LPS) for $2 \mathrm{~d}$, and analyzed using flow cytometry. Data are representative of four experiments. ${ }^{*} p<0.05$ and ${ }^{* *} p<0.01$ compared to control.

\section{Quantitative polymerase chain reaction}

Purified Tregs are prepared for cDNA for quantitative real-time polymerase chain reaction (Takara, Shiga, Japan) as described previously [21]. The relative amount for the $\beta$-actin signals was calculated as $2^{-\Delta \mathrm{Ct}} \times 10^{2}$. The specific primer sequences were synthesized by Bioneer (Daejeon, Korea). $\beta$-actin (forward CGTGAAAAGATGACCCAGATCA) (reverse TGGTACGACCAGAGGCATACAG), IL-2 (forward TCTGCGGCATGTTCTGGATTT) (reverse ATGTGTTGTCAGAGCCCTTTAG), TGF-beta (forward CCGCAACAACGCCATCTATG) (reverse CCCGAATGTCTGACGTATTGAAG), CTLA4 (forward AGTGGGCTTCCTAGATTACCC) (reverse ATGGTGAGGTTCACTCTGCTT).

\section{RESULTS}

\section{Effects of ginsenoside Rp1 on lymphocytes}

To determine the effects of G-Rp1 on lymphocyte

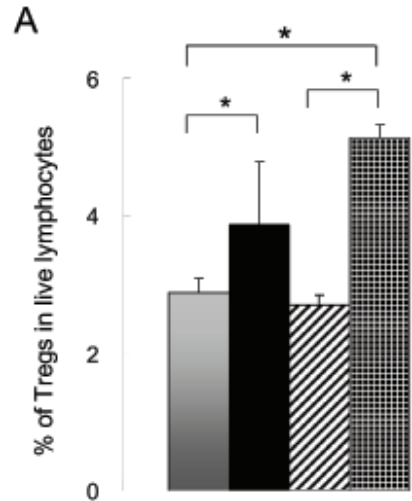

Before DC depletion
LPS G-Rp1
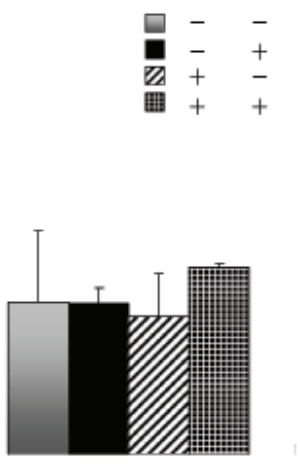

After DC depletion
B

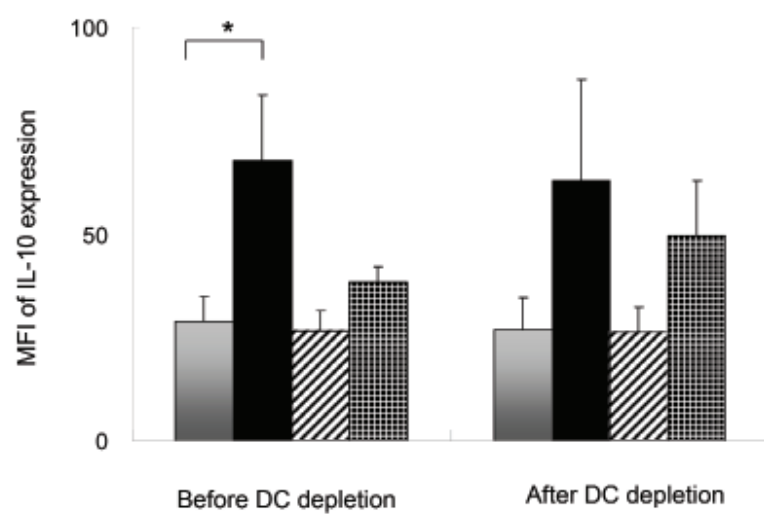

Fig. 2. Effects of dendritic cells (DCs) on regulatory $T$ cell (Treg) number and IL-10 secretion with or without ginsenoside Rp1 (G-Rp1) and lipopolysaccharide (LPS). DCs were depleted using CD11c magnetic beads and Tregs were cultured with or without $20 \mu \mathrm{M}$ G-Rp1 and/or $1 \mu \mathrm{g} / \mathrm{mL}$ LPS before and after DC depletion for $2 \mathrm{~d}$. (A) Percentage of Tregs in live lymphocytes. (B) Mean fluorescence intensity (MFI) of IL-10 expression in Tregs. These results are average of three separate experiments. Error bar shows the standard deviation. ${ }^{*} p<0.05$ compared to control.

number and activation and the optimal concentration of this agent to use to influence cell activation, splenocytes from mouse spleens were cultured with 5, 10, or $20 \mu \mathrm{M}$ G-Rp1 for $2 \mathrm{~d}$ (Fig. 1A). The relative cell numbers of helper $\mathrm{T}(\mathrm{Th})$ cells, cytotoxic $\mathrm{T}(\mathrm{Tc})$ cells, and B cells were decreased in the presence of G-Rp1. In particular, the number of Tc cells was decreased in a dose dependent manner: $17.4 \%$ with control incubation, $16.1 \%$ with $5 \mu \mathrm{M}$ G-Rp1, $12.0 \%$ with $10 \mu \mathrm{M}$ G-Rp1, and $6.6 \%$ with $20 \mu \mathrm{M}$ G-Rp1. In contrast, the number of DCs was dramatically increased in a dose-dependent manner: 6.7\% with control incubation, $9.8 \%$ with $5 \mu \mathrm{M}$ G-Rp1, $15.8 \%$ with $10 \mu \mathrm{M}$ G-Rp1, and $22.7 \%$ with $20 \mu \mathrm{M}$ G-Rp1. The number of Tregs was slightly increased from $2.9 \%$ with control treatment to $3.9 \%$ with $20 \mu \mathrm{M}$ G-Rp1. Because 
treatment with $20 \mu \mathrm{M}$ G-Rp1 showed the biggest effect on lymphocytes without accompanying cytotoxicity, further experiments were performed with $20 \mu \mathrm{M}$ G-Rp1.

\section{Effects of ginsenoside-Rp1 on inflammation}

To investigate the effects of G-Rp1 on inflammation, splenocytes were cultured in the presence of G-Rp1 with or without LPS (Fig. 1B). The relative cell numbers of Th and Tc cells, DCs, and Tregs cultured with LPS alone were decreased, but Th cells, DCs, and Tregs were increased in cultures with LPS and G-Rp1. In particular, the relative cell number of DCs was increased 7.5-fold from $3.3 \%$ in cells cultured with LPS alone to $24.9 \%$ in cells cultured with LPS and G-Rp1. In addition, the number of Tregs was also increased from $2.7 \%$ in cells cultured with LPS alone to $5.1 \%$ in cells cultured with LPS and G-Rp1, suggesting that DCs stimulated with LPS through Toll-like receptor 4 affect the cell number and activation of Tregs.

\section{Role of dendritic cells in regulatory $\mathrm{T}$ cell activa- tion}

Because the number of Tregs was increased along with DCs, the effects of LPS-activated DCs on the cell

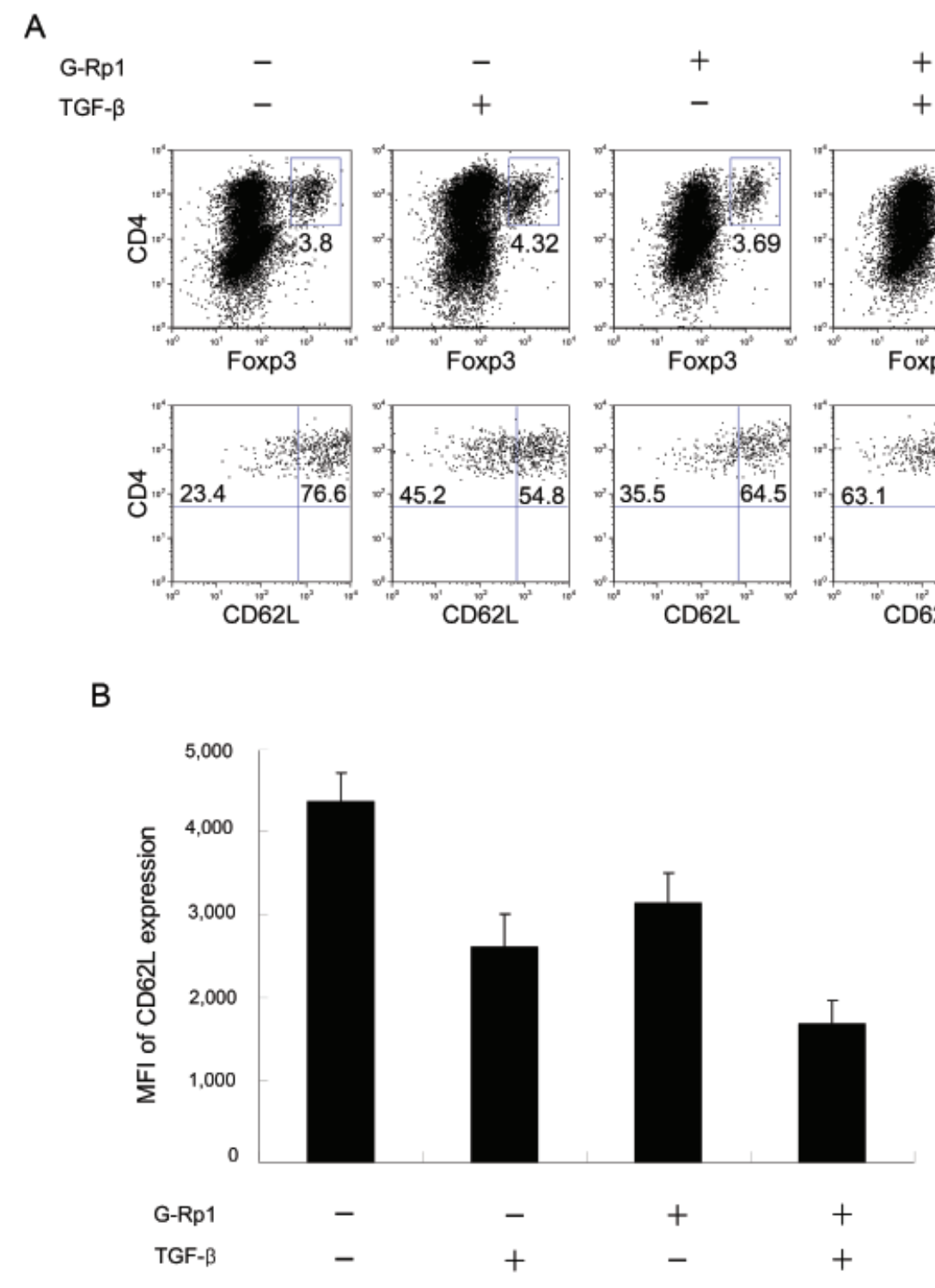

Fig. 3. Expression of CD62L on Tregs stimulated with TGF- $\beta$ and/or ginsenoside Rp1 (G-Rp1). Activated Splenocytes on anti-CD3 antibodycoated plates were cultured with or without $2 \mathrm{ng} / \mathrm{mL}$ TGF- $\beta$ for $3 \mathrm{~d}$ and then $20 \mu \mathrm{M}$ G-Rp1 for $2 \mathrm{~d}$. (A) Flow cytometry analysis of culture cells costained with $\mathrm{CD} 4$ and Foxp3. Gated $\mathrm{CD} 4^{+} \mathrm{Foxp}^{+}$cells were then stained for CD62L. Numbers in quadrants indicate percentage of $\mathrm{CD} 62 \mathrm{~L}^{\text {low }}$ cells and $\mathrm{CD} 62^{\text {high }}$ cells. (B) Mean fluorescence intensity (MFI) of CD62L expression in regulatory $\mathrm{T}$ cells. The results are average of three separate experiments. 
number and activation of Tregs were examined. For this examination, DC-depleted splenocytes were cultured with G-Rp1 and/or LPS, and Treg number and activation were analyzed (Fig. 2A). The increase in Tregs cultured with G-Rp1 was abrogated in the absence of DCs. Furthermore, Tregs stimulated with G-Rp1 and LPS in the absence of DCs did not increase. To determine whether DCs affect IL-10 expression in Tregs, IL-10 expression was measured by flow cytometry (Fig. 2B). Mean fluorescence intensity (MFI) of IL-10 expression in Tregs was increased by 2.3 -fold from 28.9 to 67.8 following incubation with G-Rp1, and this increase remained in the absence of DCs.

\section{Downregulation of CD62L expression on regula- tory $\mathrm{T}$ cells by ginsenoside Rp 1}

Although the number of Tregs following incubation with G-Rp1 was increased, it was not certain whether the increase in Tregs is due to induction of Foxp3 or conversion of existing Tregs into memory type Tregs. To investigate this, Tregs were cultured with TGF- $\beta$, which is required for the conversion of naive $\mathrm{T}$ cells into Tregs [22], and the expression of CD62L, which is highly expressed on naive cells was analyzed (Fig. 3). Treatment with TGF- $\beta$ increased $\mathrm{CD} 4{ }^{+}$Foxp ${ }^{+}$Tregs from $3.8 \%$ to $4.32 \%$ (Fig. $3 \mathrm{~A}$ ), and treatment with TGF- $\beta$ and G-Rp1 increased the percentage of $\mathrm{CD} 4{ }^{+} \mathrm{Foxp}^{+}$Tregs to $4.48 \%$. The Tregs induced by TGF- $\beta$ and G-Rp1 downregulated CD62L expression from 4364 MFI in the absence of TGF- $\beta$ and G-Rp1 to 1675 MFI in the presence of TGF- $\beta$ and G-Rp1 (Fig. 3B). This result indicates that the increase in Tregs was due to the increase of Treg survival and the conversion of memory type Tregs rather than the generation of new Tregs.

\section{Anti-inflammatory effects of ginsenoside Rp1 in vivo}

To address whether G-Rp1 has an anti-inflammatory effect in vivo and whether DCs and Tregs are involved in this effect, cells from mice that had been fed G-Rp1 for $7 \mathrm{~d}$ and subsequently injected with LPS were compared with those from mice that had not. Firstly, DCs from mice fed G-Rp1 displayed increased expression of the co-stimulatory molecules CD80 and CD86 (Fig. 4). CD80 expression was increased by 3.89 -fold compared to that in the control group and by 1.41-fold compared to that in the mice that received only LPS. CD86 expression was increased 4.63-fold and 1.37-fold compared to
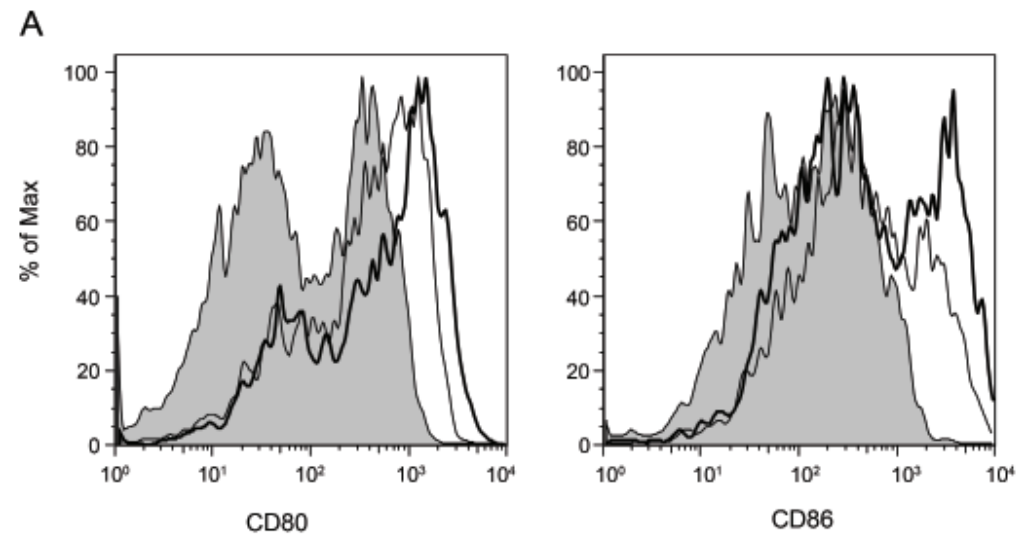

$$
\begin{aligned}
& \text { Control } \\
& - \text { LPS } \\
& - \text { G-Rp1+LPS }
\end{aligned}
$$

B
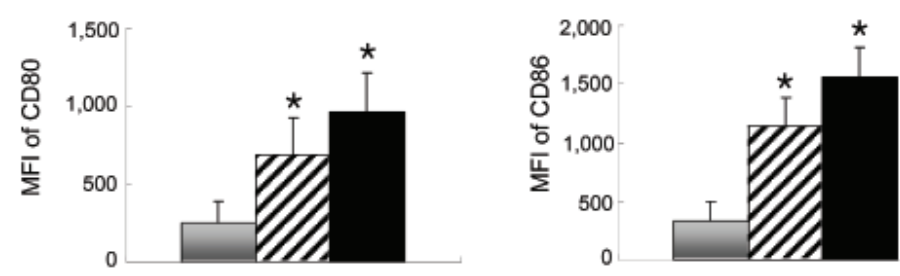
$\square$ Control
$\square$ LPS
G-Rp1+LPS

Fig. 4. Effects of ginsenoside Rp1 (G-Rp1) on dendritic cell (DC) activation in vivo. Mice that had been fed G-Rp1 for $7 \mathrm{~d}$ and subsequently injected with lipopolysaccharide (LPS) were compared with those from mice that had not. The expression of CD80 and CD86 on CD11c DCs was analyzed by flow cytometry. (A) Histogram of CD80 and CD86 expression on CD11 ${ }^{+}$DCs from phosphate-buffered saline-injected control (tinted line), LPS-injected (thin line), and G-Rp1-fed LPS-injected (thick line) mice. (B) Mean fluorescence intensity (MFI) of CD80 and CD86 expression on CD11 ${ }^{+}$DCs from control (grey bar), LPS-injected (hatched bar), and G-Rp1-fed LPS-injected (black bar) DCs. The histrograms are representatives and the MFI analyses are average of three separate experiments. ${ }^{*} p<0.05$ compared to control. 

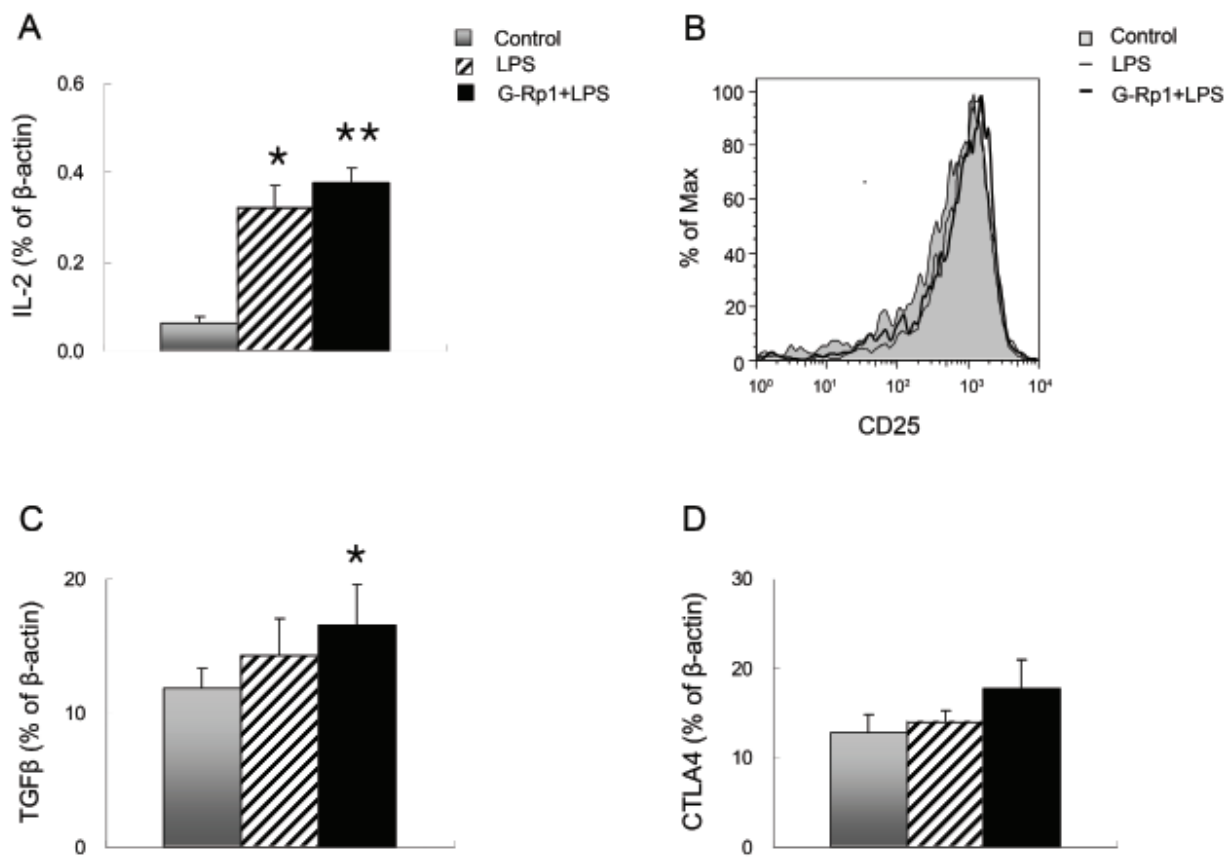

Fig. 5. Effects of ginsenoside Rp1 (G-Rp1) on IL-2, IL-2Ra, CTLA4, and TGF- $\beta$ expression in vivo. Mice that had been fed G-Rp1 for $7 \mathrm{~d}$ and subsequently injected with lipopolysaccharide (LPS) were compared with those from mice that had not. (A) IL-2 expression in splenocytes from control (grey bar), LPS-injected (hatched bar), and G-Rp1-fed LPS-injected (black bar) mice. mRNA expressions of IL-2 was normalized to $\beta$ actin signal. (B) Histogram of IL-2Ra (CD25) on regulatory T cells (Tregs) from phosphate-buffered saline-injected control (tinted line), LPSinjected (thin line), and G-Rp1-fed LPS-injected (thick line) mice. mRNA expressions of TGF- $\beta$ (C) and CTLA4 (D) in Tregs from control (grey bar), LPS-injected (hatched bar), and G-Rp1-fed LPS-injected (black bar) mice. The results are average of three separate experiments. ${ }^{*} p<0.05$ and ${ }^{* *} p<0.01$ compared to control.

that in the control and LPS-injected groups, respectively. In addition, orally-administered G-Rp1 resulted in increased IL-2 production (Fig. 5A). IL-2 was increased in this group by 6.05 -fold compared with that in the control group and 1.18-fold compared with that in the LPSinjected group. IL-2R $\alpha$ (CD25) expression on Tregs was not altered in any of the conditions (Fig. 5B). In all of the conditions, Tregs expressed high levels of IL-2R $\alpha$. In comparison, Tregs from mice fed G-Rp1 upregulated the expression of TGF- $\beta$ and CTLA4, which binds to CD80 and CD86, suggesting that Tregs had increased immune suppression activity (Fig. 5C, D).

\section{Activated $\mathrm{T}$ cells were decreased by ginsenoside Rp1 administration followed by lipopolysaccha- ride injection}

Although LPS-injected mice showed increased numbers of all lymphocytes, including Th and Tc cells, B cells, and DCs, mice that were fed G-Rp1 exhibited a smaller increase in these cell numbers (Fig. 6). In Fig. 6, cell number of each population in the control group was set up as $100 \%$. In particular, when the cell numbers were compared with LPS-injected mice, the numbers of Th

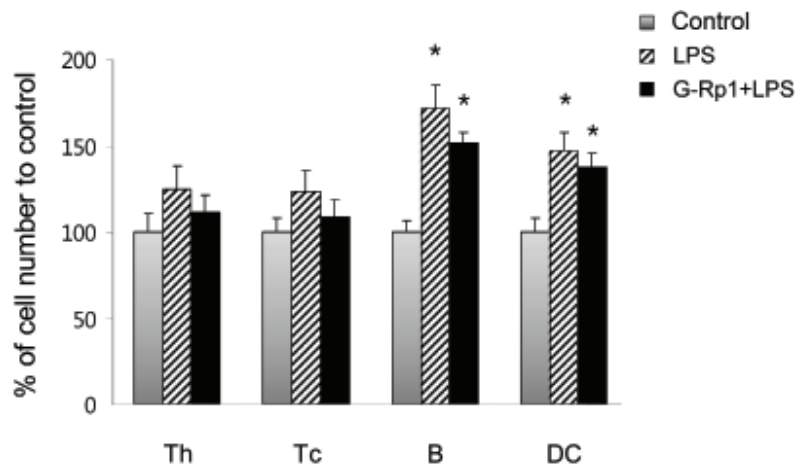

Fig. 6. Comparison of cell numbers of helper $T(T h)$, cytotoxic $T$ (TC), B and dendritic cells (DCs) from control (grey bar), lipopolysaccharide (LPS)-injected (hatched bar), and ginsenoside Rp1 (G-Rp1)fed LPS-injected (black bar) mice. Absolute cell numbers of each population were analyzed using counting beads and flow cytometry and compared with control $(100 \%)$. The results are average of three separate experiments. ${ }^{*} p<0.05$ compared to control.

and Tc cells induced by LPS injection following G-Rp1 administration were decreased from $125 \%$ to $112 \%$ and from $123 \%$ to $109 \%$, respectively; however, the numbers of B cells and DCs induced by LPS injection following G-Rp1 administration were slightly decreased from $172 \%$ to $152 \%$ and from $147 \%$ to $138 \%$, respectively. 


\section{DISCUSSION}

In previous study, Kim et al reported ginseng saponin inhibits the production of proinflammatory cytokines [23]. Here, we demonstrate the anti-inflammatory effects of G-Rp1 via activation of DCs and Tregs in vitro and in vivo.

When splenocytes were cultured with G-Rp1, DCs increased remarkably, whereas other immune cell populations decreased in a dose-dependent manner. Tregs were slightly increased in the presence of G-Rp1. This effect was enhanced when the cells were stimulated with LPS, which mimics pathological changes that occur during inflammation. LPS binds to Toll-like receptor 4 on DCs and upregulates co-stimulatory molecules, such as CD80 and CD86, on DCs. CD80 and CD86 bind to either CD28 or CTLA4 with higher affinity than CD28 [24]. In the absence of G-Rp1, LPS stimulates DCs and induces strong inflammatory responses. However, in the presence of G-Rp1, LPS stimulation increased DC and Treg numbers to a greater extent, suggesting that the increase in DCs may affect Treg numbers and function in LPS-induced inflammation. This notion was confirmed as depletion of DC prevented the increase in Treg numbers. Although IL-10 expression by Tregs was not diminished in the absence of DCs, the total amount of IL-10 was decreased as Treg numbers were not increased in this situation. The increase in Tregs by activated DCs stemmed from the conversion of $\mathrm{CD} 62 \mathrm{~L}^{\text {low }}$ memory Tregs rather than from the generation of new Tregs.

Mice that were fed G-Rp1 exhibited greater activation of DCs and Tregs. In these mice, DCs upregulated CD80 and CD86 expression, and Tregs expressed higher levels of CTLA4 and TGF- $\beta$ than cells from mice that were not fed G-Rp1. This result indicates that increased expression of CTLA4 and TGF- $\beta$ by Tregs suppresses LPS-induced inflammatory responses. In agreement with this conclusion, mice that were fed G-Rp1 followed by LPS injection showed decreased immune cell numbers, especially Th and Tc cell numbers.

Because the inflammatory responses can lead to a variety of diseases, such as septic shock, autoimmunity, and cancers, control of inflammation is paramount. Here, we report that the immune suppression function by Tregs is augmented by oral administration of G-Rp1 and that this function occurs via DC activation. We therefore suggest that orally administrated G-Rp1 may help to prevent inflammatory diseases.

\section{ACKNOWLEDGEMENTS}

This research was supported by Basic Science Research Program through the National Research Foundation of Korea (NRF) funded by the Ministry of Education, Science and Technology (NRF-2011-0024205).

\section{REFERENCES}

1. Wang W, Zhao Y, Rayburn ER, Hill DL, Wang H, Zhang R. In vitro anti-cancer activity and structure-activity relationships of natural products isolated from fruits of Panax ginseng. Cancer Chemother Pharmacol 2007;59:589-601.

2. Tode T, Kikuchi Y, Kita T, Hirata J, Imaizumi E, Nagata I. Inhibitory effects by oral administration of ginsenoside $\mathrm{Rh} 2$ on the growth of human ovarian cancer cells in nude mice. J Cancer Res Clin Oncol 1993;120:24-26.

3. Kim HS, Lee EH, Ko SR, Choi KJ, Park JH, Im DS. Effects of ginsenosides $\mathrm{Rg} 3$ and $\mathrm{Rh} 2$ on the proliferation of prostate cancer cells. Arch Pharm Res 2004;27:429-435.

4. Oh M, Choi YH, Choi S, Chung H, Kim K, Kim SI, Kim DK, Kim ND. Anti-proliferating effects of ginsenoside Rh2 on MCF-7 human breast cancer cells. Int J Oncol 1999; $14: 869-875$.

5. Shin JY, Lee JM, Shin HS, Park SY, Yang JE, Cho SK, Yi TH. Anti-cancer effect of ginsenoside F2 against glioblastoma multiforme in xenograft model in SD rats. J Ginseng Res 2012;36:86-92.

6. Park MH, Park TY, inventor. Treatment and prevention of cancer with new ginsenoside derivatives. PCT/ KR2005/001006. 2005 Jul 4.

7. Kumar A, Kumar M, Panwar M, Samarth RM, Park TY, Park MH, Kimura H. Evaluation of chemopreventive action of ginsenoside Rp1. Biofactors 2006;26:29-43.

8. Park TY, Park MH, Shin WC, Rhee MH, Seo DW, Cho JY, Kim HM. Anti-metastatic potential of ginsenoside Rp1, a novel ginsenoside derivative. Biol Pharm Bull 2008;31:1802-1805.

9. Kim BH, Lee YG, Park TY, Kim HB, Rhee MH, Cho JY. Ginsenoside Rp1, a ginsenoside derivative, blocks lipopolysaccharide-induced interleukin-1beta production via suppression of the NF-kappaB pathway. Planta Med 2009;75:321-326.

10. Shen T, Lee JH, Park MH, Lee YG, Rho HS, Kwak YS, Rhee MH, Park YC, Cho JY. Ginsenoside Rp1, a ginsenoside derivative, blocks promoter activation of iNOS and COX-2 genes by suppression of an IKKbmediated NF-kB pathway in HEK293 cells. J Ginseng Res 2011;35:200-208.

11. Bettelli E, Das MP, Howard ED, Weiner HL, Sobel RA, 
Kuchroo VK. IL-10 is critical in the regulation of autoimmune encephalomyelitis as demonstrated by studies of IL-10- and IL-4-deficient and transgenic mice. J Immunol 1998;161:3299-3306.

12. Bettelli E, Nicholson LB, Kuchroo VK. IL-10, a key effector regulatory cytokine in experimental autoimmune encephalomyelitis. J Autoimmun 2003;20:265-267.

13. Zheng Y, Rudensky AY. Foxp3 in control of the regulatory T cell lineage. Nat Immunol 2007;8:457-462.

14. Feuerer M, Hill JA, Mathis D, Benoist C. Foxp3+ regulatory $\mathrm{T}$ cells: differentiation, specification, subphenotypes. Nat Immunol 2009;10:689-695.

15. Rubtsov YP, Rasmussen JP, Chi EY, Fontenot J, Castelli L, Ye X, Treuting P, Siewe L, Roers A, Henderson WR $\mathrm{Jr}$ et al. Regulatory $\mathrm{T}$ cell-derived interleukin-10 limits inflammation at environmental interfaces. Immunity 2008;28:546-558.

16. Banchereau J, Steinman RM. Dendritic cells and the control of immunity. Nature 1998;392:245-252.

17. Cella M, Sallusto F, Lanzavecchia A. Origin, maturation and antigen presenting function of dendritic cells. Curr Opin Immunol 1997;9:10-16.

18. Darrasse-Jeze G, Deroubaix S, Mouquet H, Victora GD, Eisenreich T, Yao KH, Masilamani RF, Dustin ML, Rudensky A, Liu K et al. Feedback control of regulatory $\mathrm{T}$ cell homeostasis by dendritic cells in vivo. J Exp Med
2009;206:1853-1862.

19. Siepmann K, Biester S, Plskova J, Muckersie E, Duncan $\mathrm{L}$, Forrester JV. CD4 ${ }^{+} \mathrm{CD} 25^{+} \mathrm{T}$ regulatory cells induced by LPS-activated bone marrow dendritic cells suppress experimental autoimmune uveoretinitis in vivo. Graefes Arch Clin Exp Ophthalmol 2007;245:221-229.

20. Kim MY, Anderson G, White A, Jenkinson E, Arlt W, Martensson IL, Erlandsson L, Lane PJ. OX40 ligand and CD30 ligand are expressed on adult but not neonatal CD4+CD3- inducer cells: evidence that IL-7 signals regulate CD30 ligand but not OX40 ligand expression. J Immunol 2005;174:6686-6691.

21. Kim S, Han S, Kim MY. Effects of interleukin-15 on human $\mathrm{CD}^{-} \mathrm{CD}^{-} 17^{+} \mathrm{CD}^{-} 6^{-} \mathrm{OX}^{-} \mathrm{CL}^{+}$cell differentiation. Hum Immunol 2010;71:745-750.

22. Kretschmer K, Apostolou I, Hawiger D, Khazaie K, Nussenzweig MC, von Boehmer H. Inducing and expanding regulatory $\mathrm{T}$ cell populations by foreign antigen. Nat Immunol 2005;6:1219-1227.

23. Kim S, Shim S, Choi DS, Kim JH, Kwon YB, Kwon J. Modulation of LPS-stimulated astroglial activation by ginseng total saponins. J Ginseng Res 2011;35:80-85.

24. Van der Merwe PA, Bodian DL, Daenke S, Linsley P, Davis SJ. CD80 (B7-1) binds both CD28 and CTLA4 with a low affinity and very fast kinetics. J Exp Med 1997;185:393-403. 